\title{
ERK-1 MAP Kinase Prevents TNF-Induced Apoptosis Through Bad Phosphorylation and Inhibition of Bax Translocation in HeLa Cells
}

\author{
Bruna Pucci, ${ }^{1}$ Manuela Indelicato, ${ }^{1}$ Valentina Paradisi, ${ }^{2}$ Valentina Reali, ${ }^{2}$ Laura Pellegrini, ${ }^{2}$ \\ Michele Aventaggiato, ${ }^{2}$ Natalie 0. Karpinich, ${ }^{3}$ Massimo Fini, ${ }^{1}$ Matteo A. Russo, ${ }^{1,2}$ \\ John L. Farber, ${ }^{4}$ and Marco Tafani ${ }^{1 *}$ \\ ${ }^{1}$ Department of Cellular and Molecular Pathology, IRCCS San Raffaele Pisana, Rome, Italy \\ ${ }^{2}$ Department of Experimental Medicine and Pathology, Sapienza University, Rome, Italy \\ ${ }^{3}$ Department of Genetics, University of North Carolina, Chapel Hill, North Carolina \\ ${ }^{4}$ Department of Pathology, Thomas Jefferson University, Philadelphia, Pennsylvania
}

\begin{abstract}
Extracellular signal-regulated kinase (ERK) 1/2 signaling is involved in tumor cell survival through the regulation of Bcl-2 family members. To explore this further and to demonstrate the central role of the mitochondria in the ERK1/2 pathway we used the HeLa cellular model where apoptosis was induced by tumor necrosis factor (TNF) and cycloheximide (CHX). We show that HeLa cells overexpressing ERK-1 displayed resistance to TNF and CHX. HeLa cells overexpressing a kinase-deficient form of ERK-1 (K71R) were more sensitive to TNF and CHX. In the ERK-1 cells, Bad was phosphorylated during TNF + CHX treatment. In the HeLa wt cells and in the K71R clones TNF and CHX decreased Bad phosphorylation. ERK-1 cells treated with TNF and CHX did not release cytochrome $c$ from the mitochondria. By contrast, HeLa wt and K71R clones released cytochrome $c$. Bax did not translocate to the mitochondria in ERK-1 cells treated with TNF + CHX. Conversely, HeLa wt and K71R clones accumulated Bax in the mitochondria. In the HeLa wt cells and in both ERK-1 transfectants Bid was cleaved and accumulated in the mitochondria. The caspase-8 inhibitor IETD-FMK and the mitochondrial membrane permeabilization inhibitor bongkrekic acid (BK), partially prevented cell death by TNF + CHX. Anisomycin, a c-Jun N-terminal kinases activator, increased TNF-killing. The ERK-1 cells were resistant to TNF and anisomycin, whereas K71R clones resulted more sensitive. Our study demonstrates that in HeLa cells the ERK-1 kinase prevents TNF + CHX apoptosis by regulating the intrinsic mitochondrial pathway through different mechanisms. Inhibition of the intrinsic pathway is sufficient to almost completely prevent cell death. J. Cell. Biochem. 108: 1166-1174, 2009. (c) 2009 Wiley-Liss, Inc.
\end{abstract}

KEY WORDS: ERK-1; TNF; BAD PHOSPHORYLATION; BAX; ANISOMYCIN

$\mathrm{E}$ xtracellular signal-regulated kinase (ERK) 1/2 belong to the mitogen-activated protein kinase (MAPK) family [Ramos, 2008]. ERK1 (p44) and ERK2 (p42) are part of intracellular phosphorylation cascades from the cytoplasm to the nucleus recruited for growth factor signal transduction [Raman et al., 2007; Shaul and Seger, 2007]. In the presence of extracellular stimuli, ERK1/2 are phosphorylated and activated upon stimulation of the Ras/Raf/MEK1/ 2/ERK1/2 protein kinase cascade [Cohen et al., 2006; Kim et al., 2006; Rouleau et al., 2009]. Such phosphorylation strongly increases ERK1/
2 activity, induces its nuclear translocation, and subsequently triggers-specific gene expression [Kondoh et al., 2005]. Upon its activation, the cascade is involved in many cellular processes, such as proliferation, differentiation, migration, cell survival, and apoptosis [Lu and $\mathrm{Xu}, 2006]$. Typically, the magnitude and duration of ERK1/2 activity is determined by the balance between the activity of MEK $1 / 2$, the kinases that phosphorylate and activate ERK1/2, and the activity of phosphatases that can dephosphorylate and inactivate ERK1/2 [Kondoh and Nishida, 2007].

Abbreviations used: TNF, tumor necrosis factor; CHX, cycloheximide; ERK-1, extracellular signal-regulated kinase 1; DMEM, Dulbecco's modified Eagles minimum medium; PBS, phosphate-buffered saline; $\mathrm{Me}_{2} \mathrm{SO}$, dimethyl sulfoxide; SDS, sodium dodecyl sulfate; IETD, Z-Ile-Glu(OMe)-Thr-Asp(OMe)-FMK; BK, bongkrekic acid.

Additional Supporting Information may be found in the online version of this article.

*Correspondence to: Dr. Marco Tafani, Department of Cellular and Molecular Pathology, IRCCS San Raffaele Pisana, Rome, Italy. E-mail: marcotafani@yahoo.com

Received 17 June 2009; Accepted 11 August 2009 • DOI 10.1002/jcb.22345 • () 2009 Wiley-Liss, Inc.

Published online 23 September 2009 in Wiley InterScience (www.interscience.wiley.com). 
C-Jun N-terminal kinases (JNKs) are another subfamily of proteins belonging to the MAP kinase family. The JNKs signaling pathway can be stimulated by a variety of stresses [Johnson and Nakamura, 2007]. These include genotoxic stress, heat shock, osmotic shock, and metabolic stress, as well as pro-inflammatory cytokines, such as tumor necrosis factor (TNF) and interleukin 1beta [Weston and Davis, 2007]. JNKs phosphorylate transcription factors c-Jun, ATF-2, p53, Elk-1, which in turn regulate the expression of genes involved in cell proliferation, differentiation, and apoptosis [Dhanasekaran and Reddy, 2008; Yang and Trevillyan, 2008].

Apoptosis or programmed cell death (PCD) is a series of biochemical and morphological alterations leading to cellular dismantling through ATP-dependent mechanisms. Apoptosis can be activated by intrinsic stress, such as growth factor deprivation or cell damages, or by extrinsic mechanisms such as activation of death receptors [Strasser et al., 2000]. The ultimate decision to activate the suicide program is controlled by the Bcl-2 protein family [Gross et al., 1999]. Bcl-2 family members are divided into three subfamilies; pro-survival (Bcl-2, Bcl-xL, Bcl-w, Mcl-1, and A1), pro-apoptotic (Bax, Bak, and Bok), and BH3 pro-apoptotic (Bad, Bid, Bik, Blk, Hrk, BNIP3, and BimL) [Petros et al., 2004; Youle and Strasser, 2008]. The pro-apoptotic Bcl-2 proteins are often found in the cytosol, where they act as sensors of cellular damage or stress. During apoptosis they relocate to the surface of the mitochondria, where they induce the permeabilization of the mitochondrial membrane with the release of cytochrome $c$ and the formation of the apoptosome [Sharpe et al., 2004]. The apoptosome is a cytosolic complex formed by the interaction between cytochrome $c$, procaspase-9, and APAF-1. Clustering and activation of pro-caspase-9 leads to activation of caspase-3 [Breckenridge and Xue, 2004], which in turn promotes a number of morphological changes including cell shrinkage, chromatin condensation, DNA fragmentation, and plasma membrane blebbing [Orrenius, 2004].

The present study investigates the role of ERK-1 in the cell killing of HeLa cells by TNF and cycloheximide (CHX). We show that overexpression of ERK-1 reduces TNF toxicity through phosphorylation of the pro-apoptotic protein Bad and inhibition of the mitochondria permeability transition (MPT). Overexpression of a kinase-deficient ERK-1 (K71R) greatly increased cell killing by TNF and CHX. Finally, cell killing could be completely prevented by simultaneously inhibiting both caspase-8 activity and MPT induction.

\section{MATERIALS AND METHODS}

\section{CELL LINE}

HeLa cells (human cervix adenocarcinoma, ATCC-CC-1, Rockville, MD) were maintained in $25-\mathrm{cm}^{2}$ polystyrene flasks (Corning Costar Corp., Oneonta, NY) with $5 \mathrm{ml}$ of Dulbecco's modified Eagle's Minimum medium (DMEM; Mediatech, Inc., Hedron, VA) containing $100 \mathrm{U} / \mathrm{ml}$ penicillin, $0.1 \mathrm{mg} / \mathrm{ml}$ streptomycin, and $10 \%$ heatinactivated fetal bovine serum. The cells were incubated under an atmosphere of 95\% air and 5\% $\mathrm{CO}_{2}$. For all experiments, HeLa cells were plated at a density of 40,000 cells $/ \mathrm{cm}^{2}$ in complete DMEM.
Following overnight incubation, the cells were washed once with PBS and placed in DMEM without serum.

\section{TREATMENTS}

Human recombinant TNF-alpha (Sigma, St. Louis, MO) was dissolved in PBS and added at $10 \mathrm{ng} / \mathrm{ml}$ for the times indicated. CHX (Sigma) was dissolved in PBS and added at $10 \mu \mathrm{M}$ for the times indicated. The caspase-8 inhibitor Z-Ile-Glu(OMe)-Thr-Asp(OMe)$\mathrm{CH}_{2} \mathrm{~F}$ (IETD) (Kamiya Biomedical, Seattle, WA) was dissolved in $\mathrm{Me}_{2} \mathrm{SO}$ and added at a final concentration of $50 \mu \mathrm{M}$. In all cases, when diluted to a final concentration of $0.1 \%(\mathrm{v} / \mathrm{v}), \mathrm{Me}_{2} \mathrm{SO}$ had no effect on the cells or the parameters measured. Anisomycin (Enzo Life Sciences, Plymouth Meeting, PA) was dissolved in $\mathrm{Me}_{2} \mathrm{SO}$ and added to a final concentration of $10 \mu \mathrm{g} / \mathrm{ml}$. Bongkrekic acid (BK) (Merck Chemicals, Nottingham, UK) was dissolved in $2 \mathrm{~N} \mathrm{NH}_{4} \mathrm{OH}$ and added at a final concentration of $40 \mu \mathrm{M}$. $\mathrm{NH}_{4} \mathrm{OH}$ alone had no effect on the cells or the parameters measured.

\section{CELL VIABILITY ASSAY}

Cell viability was determined at the times indicated by the release of lactate dehydrogenase (LDH) into the culture medium as described previously [Pastorino et al., 1995].

\section{GENERATION OF STABLE ERK-1 TRANSFECTANTS}

HeLa cells were plated in $1.88 \mathrm{~cm}^{2}$ wells of a 24-well plate. After an overnight incubation, the cells were washed once with PBS and then transfected using Lipofectomine-PLUS (Invitrogen, Carlsbad, CA) according to the manufacturer's instructions. The cells were transfected with $0.5 \mu \mathrm{g}$ of pCDNA-Erk-1 wt and pCDNA-Erk-1 K71R (kinase-deficient form of Erk-1). The pCEP4L-Erk-1 wt and pCEP4L-Erk-1 K71R plasmids were generously provided by Dr. Melanie Cobb, University of Texas Southwestern Medical Center [Frost et al., 1994]. Erk-1 wt cDNA and Erk-1 K71R cDNA were subcloned into the pCDNA3. 1 vector (Invitrogen). After $4 \mathrm{~h}$ the cells were washed twice with PBS and placed into complete DMEM and incubated for $48 \mathrm{~h}$. The cells were then washed once with PBS and trypsinized. Four wells of Erk-1 wt transfected cells and four wells of the Erk-1 K71R transfected cells were placed respectively into two $75-\mathrm{cm}^{2}$ polystyrene flasks in complete DMEM supplemented with $860 \mu \mathrm{g} / \mathrm{ml}$ of G418 (Invitrogen). Stable transfectants were generated and cultured in $25-\mathrm{cm}^{2}$ polystyrene flasks. The overexpression of the HA-tagged Erk-1 wt and the overexpression of the HA-tagged Erk-1 K71R were confirmed by Western Blot analysis. Approximately $1 \times 10^{6}$ cells were collected and pelleted at $1,000 \mathrm{~g}\left(10 \mathrm{~min}\right.$ at $\left.4^{\circ} \mathrm{C}\right)$ and a total lysate was obtained. A sample containing $(30 \mu \mathrm{g})$ of proteins was separated by electrophoresis on a 10\% SDSpolyacrylamide gel. The gel was electroblotted onto a Trans-Blot Transfer Medium pure nitrocellulose membrane $(0.45 \mu \mathrm{m})$ (Bio-Rad, Hercules, CA). The blot was probed with an anti-HA rabbit polyclonal antibody (Y-11) (Santa Cruz Biotechnology, Santa Cruz, CA) at a dilution of 1:4,000. The membrane was incubated with a secondary anti-rabbit horseradish peroxidase-labeled antibody $(1: 20,000)$; the protein-antibody complex was visualized by enhanced chemiluminescence. 


\section{WHOLE CELL LYSATE}

After treatment the cells were harvested by scraping, pelleted by centrifugation at $1,000 \mathrm{~g}\left(10 \mathrm{~min}\right.$ at $\left.4^{\circ} \mathrm{C}\right)$, and then resuspended in 50-100 $\mu \mathrm{l}$ cell lysis buffer (20 mM Tris $\mathrm{pH} 7.4,100 \mathrm{mM} \mathrm{NaCl}, 1 \%$ Triton) with Proteinase Inhibitors (1 mM phenylmethylsulfonyl fluoride, $10 \mu \mathrm{g} / \mathrm{ml}$ leupeptin, $10 \mu \mathrm{g} / \mathrm{ml}$ aprotinin). The sample was centrifuged at $14,000 \mathrm{~g}\left(10 \mathrm{~min}\right.$ at $\left.4^{\circ} \mathrm{C}\right)$ to remove debris and unbroken cells.

\section{ISOLATION OF CYTOSOL AND MITOCHONDRIAL FRACTIONS}

Cells were plated in $75-\mathrm{cm}^{2}$ polystyrene flasks. Following treatment the cells were harvested by centrifugation at $750 \mathrm{~g}$ for $10 \mathrm{~min}$ at $4^{\circ} \mathrm{C}$. The cell pellets were resuspended in $1 \mathrm{ml}$ of $20 \mathrm{mM} \mathrm{HEPES}-\mathrm{KOH}(\mathrm{pH}$ 7.5), $10 \mathrm{mM} \mathrm{KCl}, 1.5 \mathrm{mM} \mathrm{MgCl}_{2}$, $1 \mathrm{mM}$ EDTA, $1 \mathrm{mM}$ EGTA, $1 \mathrm{mM}$ phenylmethylsulfonyl fluoride, $10 \mu \mathrm{g} / \mathrm{ml}$ leupeptin, $10 \mu \mathrm{g} / \mathrm{ml}$ aprotinin, and $250 \mathrm{mM}$ sucrose. The cells were rupture by six passages through a 26.5-gauge needle applied to a $1 \mathrm{ml}$ syringe. The disrupted cells were centrifuged at $750 \mathrm{~g}$ for $10 \mathrm{~min}$ at $4^{\circ} \mathrm{C}$. The pellet obtained was resuspended in PBS and stained with Trypan blue in order to identify unbroken cells. Over 95\% of the cells were disrupted as evidenced by their uptake of the dye. The supernatant was transferred to a high-speed centrifuge tube. Centrifugation was conducted at $10,000 \mathrm{~g}$ for $30 \mathrm{~min}$ at $4{ }^{\circ} \mathrm{C}$. The resulting mitochondrial pellet was lysed in $50 \mu \mathrm{l}$ of $20 \mathrm{mM}$ Tris, $\mathrm{pH} 7.4,100 \mathrm{mM} \mathrm{NaCl}, 1 \mathrm{mM}$ phenylmethylsulfonyl fluoride, $10 \mu \mathrm{g} / \mathrm{ml}$ leupeptin, $10 \mu \mathrm{g} / \mathrm{ml}$ aprotinin, and $1 \%$ Triton $\mathrm{X}-100$. The supernatant from the $10,000 \mathrm{~g}$ spin was centrifuged at $100,000 \mathrm{~g}$ (60 min at $4^{\circ} \mathrm{C}$ ), and the supernatant was used for preparation of cytosol. The cytosolic fraction was concentrated through a Microcon YM-10 Centrifugal Filter Device (Millipore, Milan, Italy). Protein content of the fractions was determined by the bicinchoninic acid assay (Sigma).

\section{WESTERN BLOT ANALYSIS}

Equivalent amounts of protein were electrophoresed on SDSpolyacrylamide gels. Kaleidoscope Prestained Standards (Bio-Rad) were used to determine molecular weight. The gels were electroblotted onto nitrocellulose membranes and probed with the following primary antibodies [Bax N-20 (Santa Cruz Biotechnology) 1:1,000, Bid (Biosource, Camarillo, CA), Bad C-7 (Santa Cruz Biotechnology) 1:500, PhosphoBad Ser-136 (Cell Signaling Technology, Beverly, MA) 1:500, Elk-1 and PhosphoElk-1 Ser 383 (Santa Cruz Biotechnology) 1:500, cytochrome c (BD International, Milan, Italy) 1:500, COX4 (Invitrogen) 1:1,000, actin C-2 (Santa Cruz Biotechnology) 1:400]. The membrane was incubated with a secondary anti-mouse $(1: 15,000)$ or anti-rabbit $(1: 15,000)$ horseradish peroxidase-labeled antibody. The protein-antibody complex was visualized by enhanced chemiluminescence.

\section{ISOLATION OF NUCLEAR FRACTION}

Cells $\left(2 \times 10^{6}\right)$ were plated in $100 \mathrm{~mm}$ dishes. The nuclear and cytosolic fractions were isolated using the nuclear extraction kit from Active Motif (Carlsbad, CA) following manufacturer's instructions. Briefly, following treatment, cells were scraped off the plate in a PBS/phosphatase inhibitor buffer using a rubber policeman. Cells were centrifuged $5 \mathrm{~min}$ at $500 \mathrm{rpm}$ at $4{ }^{\circ} \mathrm{C}$ and the supernatant discarded. The cell pellet was lysed in hypotonic buffer for $15 \mathrm{~min}$ in ice followed by centrifugation (30 s at $14,000 \mathrm{rpm}$ at $4^{\circ} \mathrm{C}$ ). The supernatant (cytosolic fraction) was transferred to a new tube, while the pellet (nuclear fraction) was lysed in a lysis buffer in the presence of protease inhibitors.

\section{ELISA ASSAY}

Nuclear extracts were obtained as described above and used to measure the relative amount of phosphorylated Elk-1 using the TransAM kit from Active Motif and following manufacturer's instructions. Briefly, equivalent amounts of protein from nuclear extracts were loaded on a 96-well plate coated with oligonucleotides containing consensus-binding site for Elk-1. After $1 \mathrm{~h}$ incubation at room temperature, wells were washed three times in wash buffer and incubated $1 \mathrm{~h}$ with a primary antibody against phosphorylated Elk-1 at room temperature. Subsequently, wells were washed and incubated $1 \mathrm{~h}$ with HRP-conjugated secondary antibody followed by the addition of a developing solution. A stop solution was added to block the reaction and the color intensity was read in a plate reader with a $450 \mathrm{~nm}$ filter. The color intensity in each well is proportional to the amount of the transcription factor bound to the oligonucleotides.

\section{STATISTICAL ANALYSIS}

All experiments were repeated 3-5 times. Significant differences between sets of values for control and test groups were assessed by a one-way ANOVA using a student Newman-Keuls post hoc test. A $P$ value refers to a comparison of a measured parameter in the experimental group with that of the appropriate control; significance was set at $P<0.05$.

\section{RESULTS}

\section{OVEREXPRESSION OF ERK-1 PREVENTS CELL KILLING BY $\mathrm{TNF}+\mathrm{CHX}$}

Clones of stably transfected HeLa cells were produced that overexpress either the wild-type ERK-1 protein (ERK-1) or a kinase-deficient form of ERK-1 (ERK-1 K71R) bearing a hemagglutinin tag (HA) (Fig. 1A). The sensitivity of these clones to TNF and CHX is shown in Figure 1B. Treatment of wild-type HeLa cells for $24 \mathrm{~h}$ with TNF and CHX killed 60\% of the cells. The same doses of TNF and CHX, however, killed only 25\% of the ERK-1 overexpressing HeLa cells and 74\% of the HeLa cells overexpressing the kinase-deficient form of ERK-1 (Fig. 1B). Supplementary Figure 1 shows that treatment of wild-type HeLa cells or ERK clones with TNF or CHX alone did not induce a significant increase in the number of dead cells.

A well-known ERK-1 and ERK-2 substrate is Elk-1. Elk-1 is a transcription factor that, along with Sap 1 and Net, forms the ternary complex factor (TCF) involved in regulating gene expression by DNA binding at the serum-response elements (SRE) [Buchwalter et al., 2004]. The DNA binding activity of Elk-1 is regulated through phosphorylation on serine 383 residue by ERK1/2. Therefore, we used the phosphorylation state of Elk-1 on serine 383 to measure the kinase activity of our ERK-1 clones. In particular, we used 


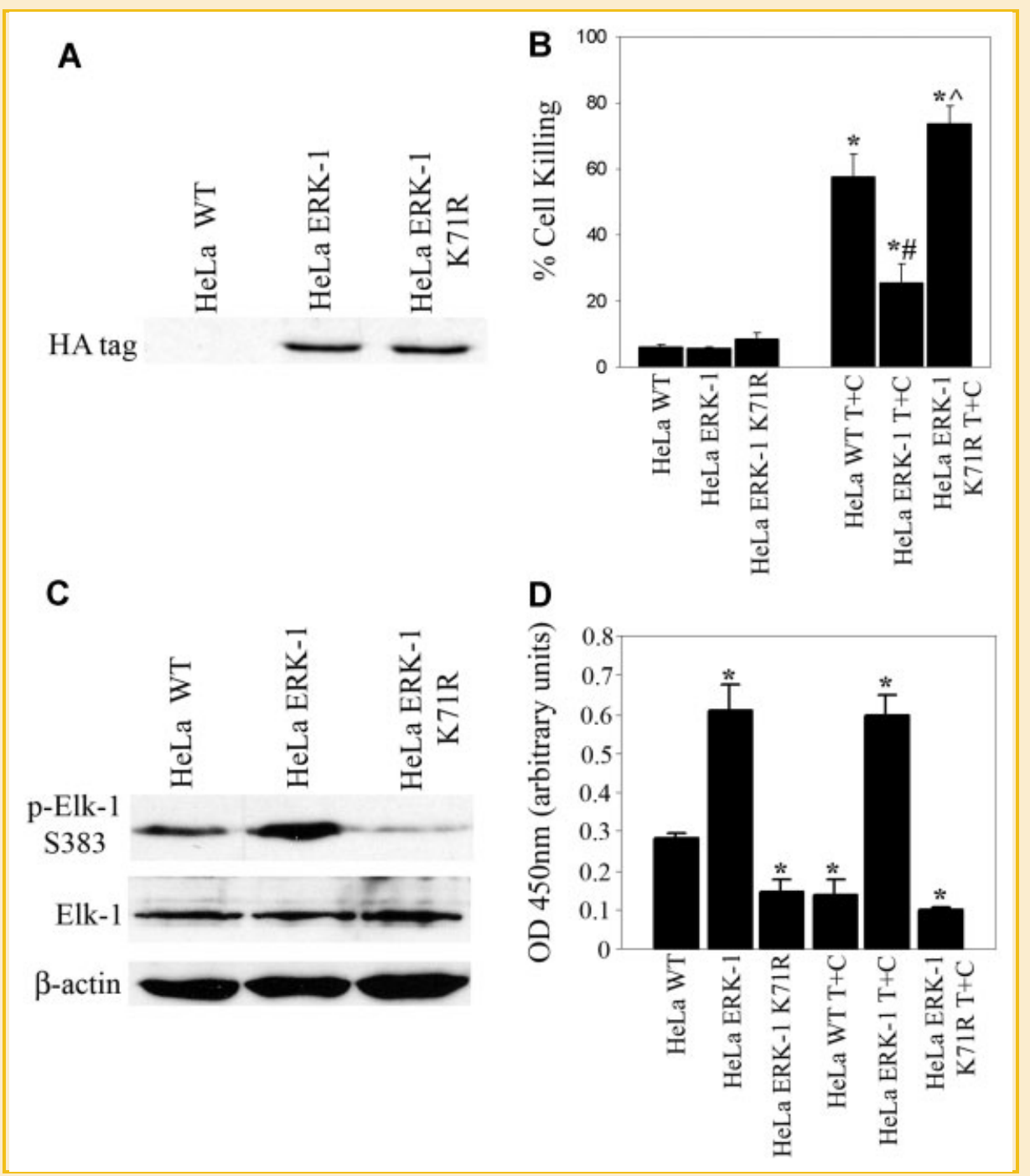

Fig. 1. TNF + CHX killing and ERK-1 activity in wild-type, ERK-1 and ERK-1 K71R HeLa cells. A: HeLa cells (ERK-1 and ERK-1 K71R) were stably transfected with an ERK-1 expression vector as described in Materials and Methods Section. Expression of ERK-1 and ERK-1 K71R was determined by Western blot analysis. WT, wild-type cells. B: Wildtype, ERK-1 and ERK-1 K71R HeLa cells were treated with TNF and CHX for $24 \mathrm{~h}$. Viability of the cells was determined by LDH release. Results are the mean \pm SD of three separate experiments. "Statistically different from the control group $(P<0.05) .{ }^{* \#}$ Statistically different from the control, HeLa WT T + C and HeLa ERK-1 K71R T $+C$ group $(P<0.05)$. ${ }^{*}$ Statistically different from the control and the HeLa WT T + C group $(P<0.05)$. C: Wild-type, ERK-1 and ERK-1 K71R HeLa cells were either left untreated or treated with TNF and CHX for $6 \mathrm{~h}$. Phosphorylation of Elk-1 on serine 383 as well as total Elk-1 content was determined by Western blot analysis. The Western blot shown is representative of three repeats. D: Nuclear fractions were obtained from wild-type, ERK-1 and ERK-1 K71R HeLa cells either left untreated or treated with TNF and CHX for 6 h. The amount of oligonucleotide-bound phosphorylated Elk-1 was measured by the ELISA-based TransAM method. Results are the mean \pm SD of three separate experiments. *Statistically different from the control wild-type group $(P<0.05)$.

to separate techniques, Western blot assay with an antibody recognizing phosphorylated Elk-1 and the Elk-1 transcription factor assay kit. Figure 1C shows that ERK-1 overexpressing cells had an increased phosphorylated Elk-1 whereas, in ERK-1 K71R cells phosphorylation of Elk-1 on Ser 383 was greatly reduced. Similarly, Figure 1D shows that DNA binding activity of phosphorylated Elk-1 was increased in ERK-1 overexpressing cells, while being significantly reduced in ERK-1 K71R cells. Finally, TNF and CHX treatment reduced Elk-1-DNA binding activity in HeLa wt and ERK-1 K71R cells but not in the ERK-1 overexpressing cells (Fig. 1D).

\section{OVEREXPRESSION OF ERK-1 PREVENTS BAD DE-PHOSPHORYLATION IN RESPONSE TO TNF + CHX}

The BH-3 only pro-apoptotic protein Bad is localized in the cytosol where it associates with the molecular chaperone protein 14-3-3 [Tzivion et al., 2001]. Such an association is achieved through the phosphorylation of Bad on serine residues [Zha et al., 1996]. Upon its phosphorylation, Bad cannot bind to the anti-apoptotic proteins Bcl-xL or Bcl-2 that can, therefore, elicit their pro-survival activity [Zha et al., 1996]. Either directly or indirectly, ERK proteins are involved in the survival pathways involving the phosphorylation of Bad [Scheid et al., 1999; Chen et al., 2008]. 


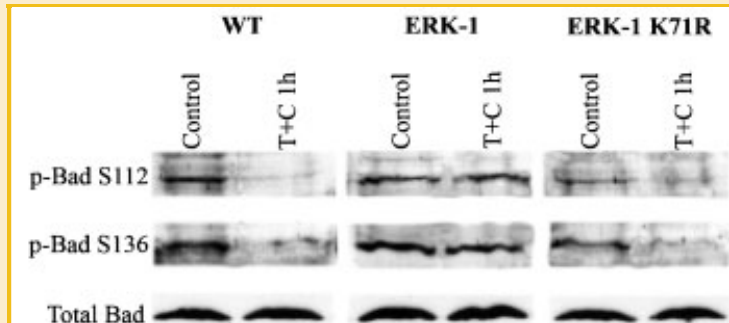

Fig. 2. Phosphorylation of Bad at Ser-112 and Ser-136 in wild-type and HeLa cells overexpressing ERK-1 or ERK-1 K71R. Wild-type, ERK-1 and ERK-1 K71R HeLa cells were treated with TNF and CHX for $1 \mathrm{~h}$. Phosphorylation of Bad at Ser-112 and Ser-136, as well as the total Bad content, was determined by Western blot analysis as described in Materials and Methods Section. The Western blot shown is representative of three repeats.

Therefore, the phosphorylation of Bad at both Ser-112 and Ser136 was studied after treating the wild-type HeLa cells and the ERK clones with TNF. As shown in Figure 2, treatment of wild-type HeLa cells with TNF and CHX for $1 \mathrm{~h}$ resulted in de-phosphorylation of Bad on both Ser-112 and Ser-136 without any change in total amount of the protein. By contrast, Bad remained phosphorylated on Ser-112 and Ser-136 in the ERK-1 overexpressing HeLa cells during the treatment with TNF and CHX for $1 \mathrm{~h}$ (Fig. 2). HeLa cells overexpressing the kinase-deficient form of ERK-1 (K71R) showed a decreased constitutive phosphorylation of Bad on both Ser-112 and Ser-136 that was completely inhibited by $1 \mathrm{~h}$ treatment with TNF and CHX (Fig. 2).

\section{OVEREXPRESSION OF ERK-1 PREVENTS CYTOCHROME $C$ RELEASE}

The release of cytochrome $c$ from the mitochondria into the cytosol is a widely used marker of mitochondrial alterations in apoptosis, including the MPT. Figure 3 shows that treatment of wild-type HeLa

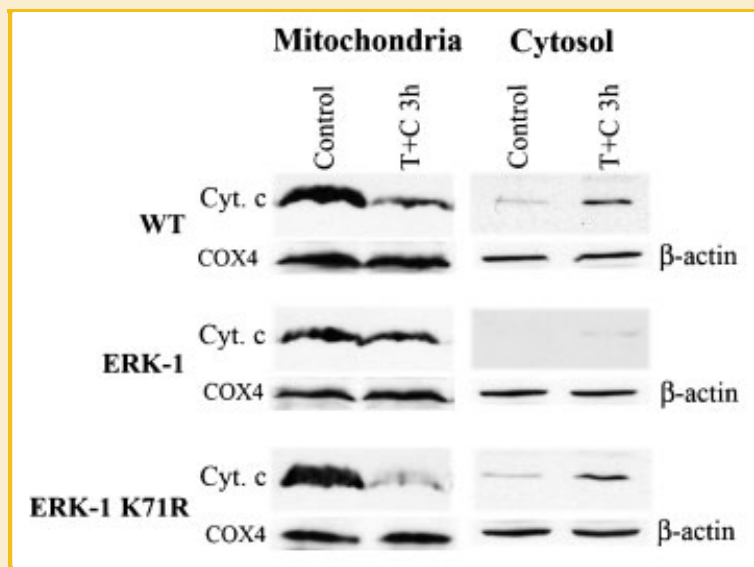

Fig. 3. Release of cytochrome $c$ from mitochondria in wild-type and HeLa cells overexpressing ERK-1 or ERK-1 K71R. Wild-type, ERK-1 and ERK-1 K71R HeLa cells were treated with TNF and $\mathrm{CHX}$ for $3 \mathrm{~h}$. The content of cytochrome $c_{\text {, }}$ $\beta$-actin, and COX4 in the cytosolic and mitochondrial subcellular fractions was determined by Western blot assay as described in Materials and Methods Section. The Western blot shown is representative of three repeats. cells with TNF and CHX produced a decrease in the mitochondrial content of cytochrome $c$ that was evident after $3 \mathrm{~h}$. The depletion of cytochrome $c$ from the mitochondrial fraction was accompanied by an increase of this protein in the cytosolic fraction (Fig. 3). A similar release of cytochrome $c$ from the mitochondria to the cytosol was observed with the HeLa cells overexpressing the kinase-deficient ERK-1 (K71R) (Fig. 3). By contrast, overexpression of ERK-1 inhibited the release of cytochrome $c$ by TNF and CHX treatment (Fig. 3). In fact, the ERK-1 overexpressing HeLa cells did not show any cytosolic accumulation of cytochrome $c$ following $3 \mathrm{~h}$ treatment with TNF and CHX (Fig. 3). Figure 3 shows that neither the content of the mitochondrial marker protein COX4 nor that of the cytosolic marker protein $\beta$-actin varied under the conditions studied.

\section{OVEREXPRESSION OF ERK-1 PREVENTS TRANSLOCATION OF BAX TO THE MITOCHONDRIA}

The pro-apoptotic protein Bax translocates from the cytosol to the mitochondria under a variety of stimuli [Pastorino et al., 1999; Tafani et al., 2001]. Figure 4 shows that treatment of wild-type HeLa cells with TNF and CHX for $3 \mathrm{~h}$ caused a redistribution of Bax from the cytosol to the mitochondria. A similar result was obtained with the HeLa cell line overexpressing the kinase-deficient ERK-1 (K71R) (Fig. 4). By contrast, when the HeLa cells overexpressing ERK-1 were treated with TNF and CHX the content of Bax did not increase in the mitochondria or decreased in the cytosol (Fig. 4). Figure 4 shows that neither the content of the mitochondrial marker protein COX4 nor that of the cytosolic marker protein $\beta$-actin varied under the conditions studied.

\section{OVEREXPRESSION OF ERK-1 DOES NOT PREVENT BID CLEAVAGE BY TNF + CHX}

The pro-apoptotic protein Bid is expressed constitutively in HeLa cells. TNF and CHX treatment for $3 \mathrm{~h}$ caused the cleavage of Bid by

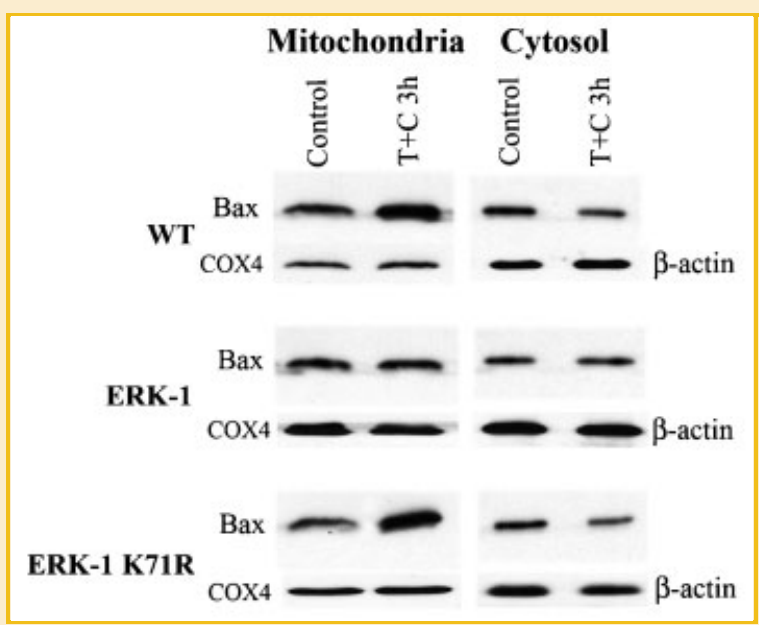

Fig. 4. Translocation of Bax from the cytosol to the mitochondria in wildtype and HeLa cells overexpressing ERK-1 or ERK-1 K71R. Wild-type, ERK-1 and ERK-1 K71R HeLa cells were treated with TNF and CHX for $3 \mathrm{~h}$. The content of Bax, $\beta$-actin, and COX4 in the cytosolic and mitochondrial subcellular fractions was determined by Western blot assay as described in Materials and Methods Section. The Western blot shown is representative of three repeats. 


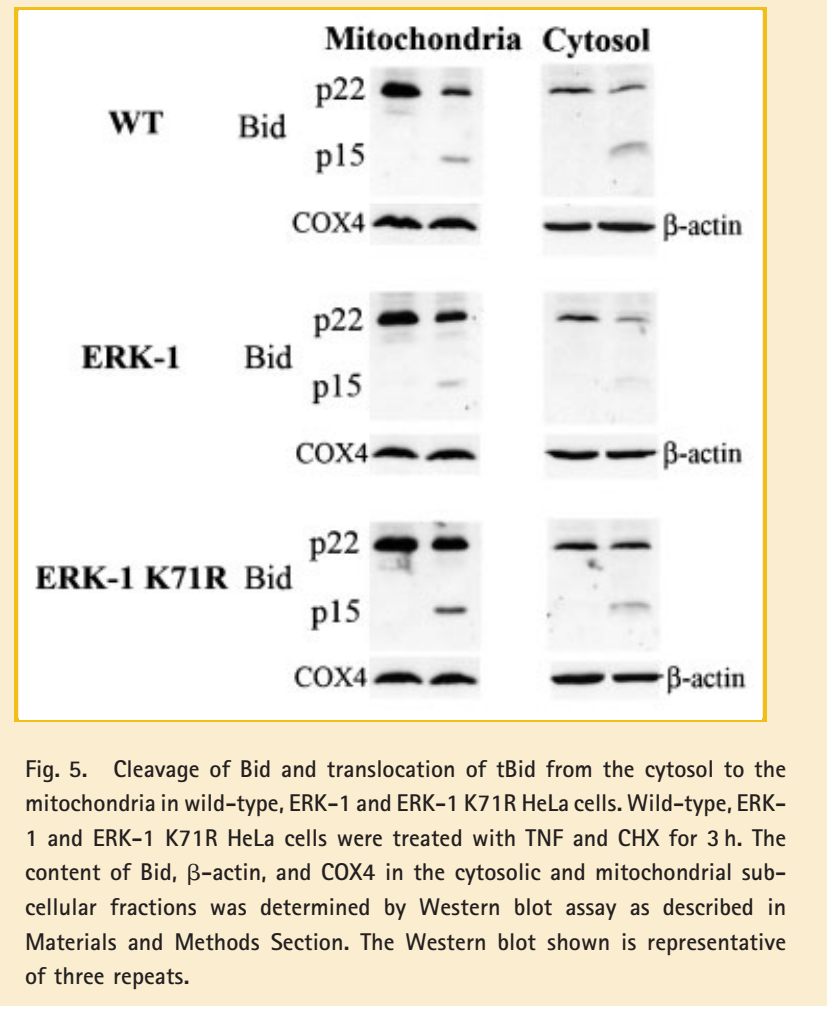

caspase-8 to form truncated Bid (tBid) that, in turn, accumulated in both the mitochondria and the cytosol (Fig. 5). Figure 5 compares the cleavage of $\mathrm{Bid}$ and the accumulation of tBid in the mitochondrial and cytosolic fractions of HeLa cell wild-type, HeLa cells overexpressing ERK-1, and HeLa cells overexpressing the kinase-deficient form of ERK-1 (K71R). Bid was cleaved and tBid accumulated in the mitochondria in both the HeLa wild-type and the HeLa ERK-1 K71R (Fig. 5). Overexpression of ERK-1 resulted in a slight inhibition of tBid accumulation in the mitochondria, probably owing to the absence of cytochrome $c$ release and caspase- 3 activation in these cells as observed in Figure 3 (Fig. 5). Figure 5 shows that neither the content of the mitochondrial marker protein COX4 nor that of the cytosolic marker protein $\beta$-actin varied under the conditions studied.

\section{OVEREXPRESSION OF ERK-1 AND INHIBITION OF CASPASE-8 COMPLETELY PREVENTS CELL KILLING BY TNF + CHX}

TNF and CHX treatment of HeLa cells activates the apoptotic program both through the death receptor extrinsic and the mitochondria-dependent intrinsic pathway [Shi and Shen, 2008]. In order to study the role of each of these two pathways, HeLa cell wild-type, HeLa cells overexpressing ERK-1 and HeLa cells overexpressing the kinase-deficient form of ERK-1 (K71R) were treated for $24 \mathrm{~h}$ with TNF $+\mathrm{CHX}$ in the presence of the caspase- 8 inhibitor IETD, the MPT inhibitor BK, or the two together. Figure 6 shows that treatment of HeLa wt cells with TNF + CHX and IETD or BK resulted in about 50\% inhibition of cell death. However, when IETD and BK were both added to the cell culture, they completely prevented cell killing. Similarly, in HeLa cells overexpressing ERK

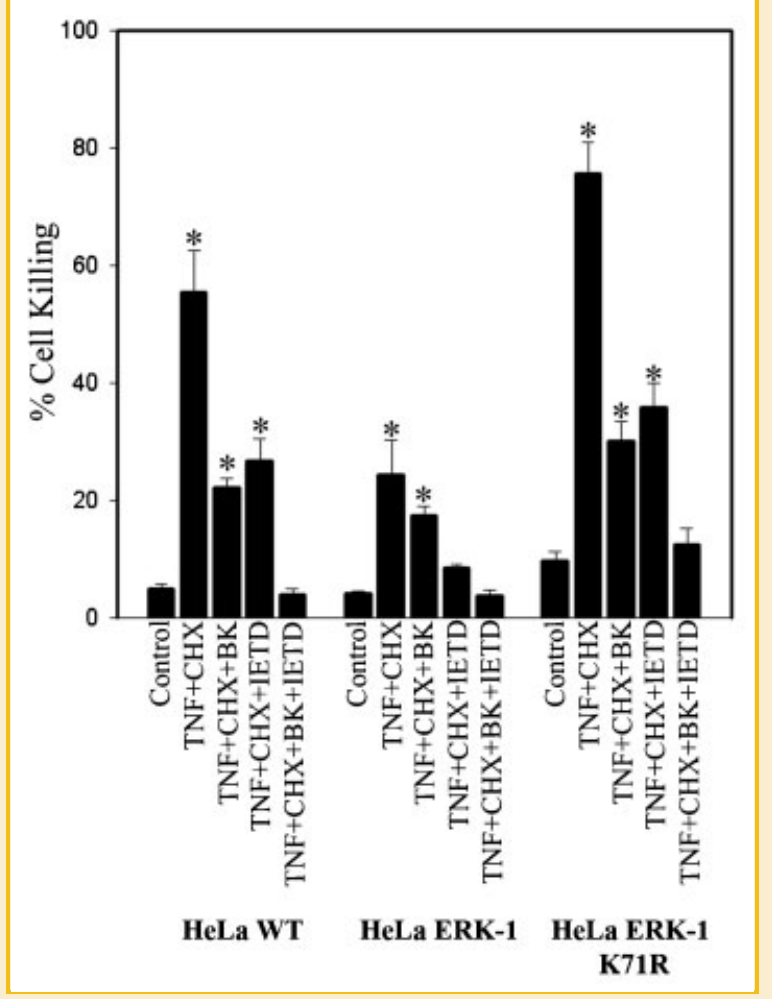

Fig. 6. Prevention by IETD and BK of the killing of wild-type, ERK-1 and ERK1 K71R HeLa cells by TNF + CHX. Wild-type, ERK-1 and ERK-1 K71R HeLa cells were treated with TNF and $\mathrm{CHX}$ for $24 \mathrm{~h}$. Viability of the cells was determined as described under Materials and Methods Section. Results are the mean \pm SD of three separate experiments. *Statistically different from the control of the corresponding group $(P<0.05)$.

K71R, IETD, or BK treatment protected 50\% of the cells from TNF toxicity. When IETD and BK were added simultaneously, the percentage of cell killing was similar to that of the control. By contrast, in HeLa cells overexpressing ERK-1 the protection afforded by the BK was minimal because the mechanism of action of this molecule (MPT inhibition) is very similar to the one obtained through ERK-1 overexpression. In fact, in these cells, IETD alone was able to reduce the extent of cell death to that of the control (Fig. 6).

\section{OVEREXPRESSION OF ERK-1 PREVENTS CELL KILLING BY TNF + ANISOMYCIN}

JNK kinases are activated in response to a variety of cell stresses [Weston and Davis, 2007]. JNK kinases pathway has been shown to be activated during apoptosis [Dhanasekaran and Reddy, 2008]. Therefore, the pro-apoptotic action of the JNK kinases can be seen as opposed to the pro-survival action of the ERK kinases. Anisomycin is a well-known JNK activator that has been shown to induce apoptosis in many cell lines. Figure 7 shows that treatment of HeLa wt cells with TNF and anisomycin for $24 \mathrm{~h}$ resulted in a 38\% cell death induction. The same dose of TNF and anisomycin, however, killed only 20\% of the cells overexpressing the ERK-1 protein and 70\% of the ERK-1 (K71R) cells (Fig. 7). Importantly, anisomycin alone did not significantly increase the percentage of cell death (Fig. 7). 


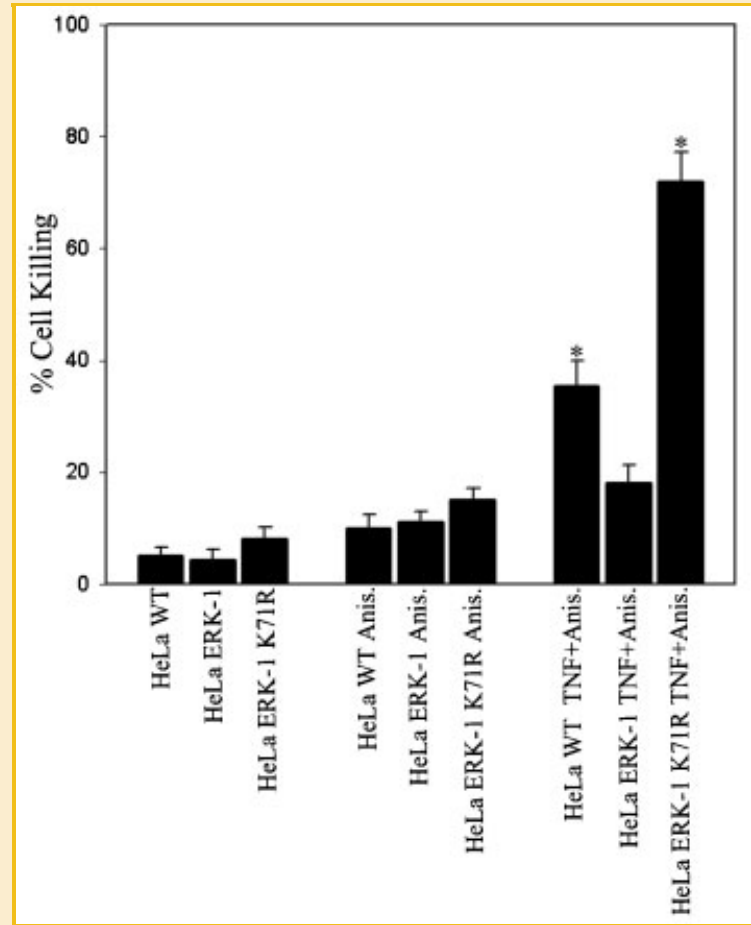

Fig. 7. TNF + anisomycin killing of wild-type, ERK-1 and ERK-1 K71R HeLa cells. Wild-type, ERK-1 and ERK-1 K71R HeLa cells were treated with TNF and anisomycin for $24 \mathrm{~h}$. Viability of the cells was determined as described under Materials and Methods Section. Results are the mean \pm SD of three separate experiments. "Statistically different from the control group $(P<0.05)$.

\section{DISCUSSION}

In a previous work from our group we have shown that HeLa cells constitute a useful cellular model to study both the growth factor and the cytotoxic effects of TNF- $\alpha$ [Pastorino et al., 1999]. Furthermore, we showed that TNF-induced phosphorylation of Bad on serine 136 by activating the phosphatidylinositide3-OH kinase (PI3K)-dependent pathway. In particular, PI3K signaling would lead to activation of the serine/threonine kinase Akt that, in turn, phosphorylates Bad on serine 136 [Pastorino et al., 1999]. We also showed that the PI3K-Akt pathway, once overexpressed, increased resistance of HeLa cells to other apoptotic stimuli such as staurosporine [Tafani et al., 2001]. Recently, also the ERK1/2 pathway has been shown to be a fundamental survival pathway that can substitute or accompany the PI3K-Akt signaling [Balmanno and Cook, 2009]. In light of our detailed knowledge of the pathways activated by TNF in the HeLa cell system, we decided to use this same cell line to study the mechanism through which ERK1/2 signaling regulates TNF toxicity. Such an approach allowed us to compare three of the main cellular pathway activated by TNF: PI3K-Akt, ERK-1, and JNK. We acknowledge the fact that our study is restricted to a single cell line, however, we also considered that so far many tumors and tumor cell lines have been shown to depend on the activation or overexpression of ERK1/2 pathway for their survival [Wu et al., 2004; Balmanno and Cook, 2009].
Herewith we show that overexpression of ERK-1 in HeLa cells prevents only the intrinsic mitochondrial-dependent apoptotic pathway activated by TNF and CHX and that such a pathway plays a major role in carrying on the apoptotic program. In particular, we showed that, during TNF and CHX treatment, HeLa ERK-1 overexpressing cells maintained Bad phosphorylated and did not show any MPT induction, as measured by mitochondrial translocation of Bax and cytochrome $c$ release. By contrast, TNF and CHX treatment of HeLa cells overexpressing a kinase-deficient ERK-1 (K$71 \mathrm{R})$ resulted in an increased mitochondrial-dependent apoptotic pathway that was preceded by Bad de-phosphorylation, intracellular redistribution of Bax, and cytochrome $c$ release.

The pro-apoptotic protein Bad has been implicated in the induction of the apoptotic pathway by several stimuli [Tafani et al., 2001; Sastry et al., 2006]. In fact, phosphorylation of Bad on serine 112 and 136 results in its cytoplasmic localization and dissociation from Bcl-xL which, in turn, can bind and inactivate the proapoptotic protein Bax [Zha et al., 1996]. Here we show that ERK-1 is involved in the phosphorylation of Bad at Ser-112 and Ser-136. Bad phosphorylation by ERK-1 allows for the inhibition of the intrinsic apoptotic pathway activated by TNF and CHX. Further studies will be necessary, however, to evaluate if the phosphoylation of Bad by ERK-1 is a direct or indirect effect. In fact, while phosphorylation of Ser-112 has been shown to depend by ERK-1, Ser-136 is believed to depend on AKT activity [Scheid et al., 1999]. The observation that Bad is de-phopshorylated on Ser-136 in HeLa cells overexpressing a kinase-deficient ERK-1, suggests that this kinase may be also involved in such a process.

The anti-apoptotic effect of ERK-1 overexpression resulted in a significant inhibition of cell death by TNF and CHX (Fig. 1). TNF and CHX treatment, however, still killed 25\% of the ERK-1 overexpressing HeLa cell. Therefore, we studied the role of the proapoptotic protein Bid. Following the activation of the extrinsic apoptotic pathway, Bid is cleaved by active caspase- 8 generating a truncated form called tBID [Li et al., 1998; Luo et al., 1998]. Both full length and truncated Bid can translocate to the mitochondria, thereby inducing the release of cytochrome $c$ and the activation of downstream caspases such as caspase-3. However, tBid can also be formed by a loop as a result of the activation of caspase- 8 by activated caspase-3. Our results show that ERK-1 overexpression can prevent such a loop without affecting the caspase-8-dependent formation of tBid. In fact, in HeLa cells overexpressing ERK-1, there was only a reduction of Bid cleavage, and some tBid still accumulated in both the cytosolic and mitochondrial subcellular fractions (Fig. 5). The role of ERK-1 in caspase-8 activation and MPT induction was demonstrated by treating HeLa cells with IETD and BK. We showed that ERK-1 controls the intrinsic apoptotic pathway by inhibiting the translocation of Bax and the induction of the MPT. However, ERK-1 did not prevent the cleavage of Bid by caspase-8 following activation of the TNF receptor. In fact, treatment of HeLa ERK-1 cells with the caspase-8 inhibitor IETD completely prevented the cell killing by TNF and CHX (Fig. 6). This same result was obtained in HeLa wt cells when both caspase-8 activation and MPT induction were inhibited by treating cells with IETD and BK (Fig. 6). Elevated levels of dually phosphorylated (active) ERK-1/ERK-2 have been reported in several human cancers, including colon, prostate, 
renal cell, and breast adenocarcinomas, as well as in head and neck squamous-cell carcinoma, glial neoplasm, and melanoma [HandraLuca et al., 2003; Zhang et al., 2004; Li and Yang, 2009]. In particular, ERK1/2 and its phosphorylated form have been shown to represent an important biological marker in breast carcinoma and gallbladder adenocarcinoma [Zhang et al., 2004; Li and Yang, 2009]. Furthermore, it is worth noting that sorafenib, a ERK1/2 inhibitor, is a drug approved for the treatment of primary kidney cancer and advanced primary liver cancer. Similarly, another ERK1/2 inhibitor, AZD6244 is entering phase II clinical trial. In this context, our study provides an important contribute to understand the role of ERK-1Bcl-2 family-mitochondria axis in HeLa cells exposed to TNF and offer a cellular model to understand the mechanism of action of newly synthesized ERK1/2 inhibitors. Furthermore, a combined approach based on recombinant ligands or mAbs for TNF and ERK1/ 2 inhibitors could prove beneficial against tumors with altered ERK1/2 signaling.

The c Jun N-terminal kinase or JNK is involved in the apoptotic pathway activated by different stimuli [Weston and Davis, 2007; Dhanasekaran and Reddy, 2008]. JNK has been shown to phosphorylate and inactivate the anti-apoptotic protein $\mathrm{Bcl}-2$, thereby inducing the MPT [Basu et al., 2000; Deng et al., 2001]. Therefore, we expected to have a similar percentage of cell killing by substituting CHX with anisomycin in the presence of TNF. In fact, TNF + CHX causes cell death by decreasing the activity of ERK-1, whereas TNF + anisomycin causes cell death by increasing the activity of JNK. Furthermore, we expected overexpression of ERK-1 to prevent cell killing induced by anisomycin. Our results show that ERK-1, by preventing the induction of MPT, can inhibit the JNK proapoptotic pathway. In fact, overexpression of ERK-1 in HeLa cells prevented the apoptotic pathway activated by the JNK agonist anisomycin. HeLa ERK-1 cells showed a significant reduction in cell death when treated with TNF and anisomycin (Fig. 7). By contrast, HeLa ERK-1 K71R cells showed an increased sensitivity to TNF and anisomycin (Fig. 7).

In HeLa cells TNF alone does not induce cell death probably because of the activation of NF-kB by ERK1/2 and AP-1 by JNK. However, when a protein synthesis inhibitor such as CHX is added, the cell killing properties of TNF prevail. Supplemental Figure 2 represents, schematically, the known survival and apoptotic events activated by TNF. The possible pathways and manipulations discussed above have been inserted in the scheme and the major players and events controlling cell survival or death evidenced in bold. In particular, our results show that activation of ERK-1 represents a fundamental event for cell survival following activation of the TNF receptor by TNF- $\alpha$. In fact, supplemental Figure 2 shows that the major targets of the intracellular events regulated by ERK-1 are the mitochondria that maintain their membrane potential status. This ERK-1 pro-survival effect overcomes pro-apoptotic situations such as JNK activation or death receptor activation and, therefore, explains the resistance of tumor cells to several pro-apoptotic stimuli.

The possible implications of our system where we overexpress a wt or kinase-deficient form of ERK-1 and observe the effects on cell survival, become clear if one considers that many tumors overexpress ERK1/2 proteins and that this correlates with tumor progression [Chen et al., 2004] or can be used as an independent prognostic predictor [Li and Yang, 2009].

\section{REFERENCES}

Balmanno K, Cook SJ. 2009. Tumour cell survival signalling by the ERK1/2 pathway. Cell Death Differ 16:368-377.

Basu A, You SA, Haldar S. 2000. Regulation of Bcl2 phosphorylation by stress response kinase pathway. Int J Oncol 16:497-500.

Breckenridge DG, Xue D. 2004. Regulation of mitochondrial membrane permeabilization by BCL-2 family proteins and caspases. Curr Opin Cell Biol $16: 647-652$.

Buchwalter G, Gross C, Wasylyk B. 2004. Ets ternary complex transcription factors. Gene 324:1-14.

Chen L, He HY, Li HM, Zheng J, Heng WJ, You JF, Fang WG. 2004. ERK1/2 and p38 pathways are required for P2Y receptor-mediated prostate cancer invasion. Cancer Lett 215:239-247.

Chen J, Volpi S, Aguilera G. 2008. Anti-apoptotic actions of vasopressin in H32 neurons involve MAP kinase transactivation and Bad phosphorylation. Exp Neurol 211:529-538.

Cohen M, Meisser A, Haenggeli L, Bischof P. 2006. Involvement of MAPK pathway in TNF-a-induced MMP-9 expression in human trophoblastic cells. Mol Hum Reprod 12:225-232.

Deng X, Xiao L, Lang W, Gao F, Ruvolo P, May WS, Jr. 2001. Novel role for JNK as a stress-activated Bcl2 kinase. J Biol Chem 276:23681-23688.

Dhanasekaran DN, Reddy EP. 2008. JNK signaling in apoptosis. Oncogene 27:6245-6251.

Frost JA, Geppert TD, Cobb MH, Feramisco JR. 1994. A requirement for extracellular signal-regulated kinase (ERK) function in the activation of AP-1 by Ha-Ras, phorbol 12-myristate 13-acetate, and serum. Proc Natl Acad Sci USA 91:3844-3848.

Gross A, McDonnell JM, Korsmeyer SJ. 1999. BCL-2 family members and the mitochondria in apoptosis. Genes Dev 13:1899-1911.

Handra-Luca A, Bilal H, Bertrand JC, Fouret P. 2003. Extra-cellular signalregulated ERK-1/ERK-2 pathway activation in human salivary gland mucoepidermoid carcinoma: Association to aggressive tumor behavior and tumor cell proliferation. Am J Pathol 163:957-967.

Johnson GL, Nakamura K. 2007. The c-jun kinase/stress-activated pathway: Regulation, function and role in human disease. Biochim Biophys Acta 1773:1341-1348.

Kim J, Lee CK, Park HJ, Kim HJ, So HH, Lee KS, Lee HM, Roh HY, Choi WS, Park TK, Kim B. 2006. Epidermal growth factor induces vasoconstriction through the phosphatidylinositol 3-kinase-mediated mitogen-activated protein kinase pathway in hypertensive rats. J Pharmacol Sci 101:135-143.

Kondoh K, Nishida E. 2007. Regulation of MAP kinases by MAP kinase phosphatases. Biochim Biophys Acta 1773:1227-1237.

Kondoh K, Torii S, Nishida E. 2005. Control of MAP kinase signaling to the nucleus. Chromosoma 114:86-91.

Li Q, Yang Z. 2009. Expression of phospho-ERK1/2 and PI3-K in benign and malignant gallbladder lesions and its clinical and pathological correlations. J Exp Clin Cancer Res 28:65.

Li H, Zhu H, Xu CJ, Yuan J. 1998. Cleavage of BID by caspase 8 mediates the mitochondrial damage in the Fas pathway of apoptosis. Cell 94:491-501.

Lu Z, Xu S. 2006. ERK1/2 MAP kinases in cell survival and apoptosis. IUBMB Life 58:621-631.

Luo X, Budihardjo I, Zou H, Slaughter C, Wang X. 1998. Bid, a Bcl2 interacting protein, mediates cytochrome c release from mitochondria in response to activation of cell surface death receptors. Cell 94:481-490.

Orrenius S. 2004. Mitochondrial regulation of apoptotic cell death. Toxicol Lett 149:19-23. 
Pastorino JG, Snyder JW, Hoek JB, Farber JL. 1995. Ca2 ${ }^{+}$depletion prevents anoxic death of hepatocytes by inhibiting mitochondrial permeability transition. Am J Physiol 268:C676-C685.

Pastorino JG, Tafani M, Farber JL. 1999. Tumor necrosis factor induces phosphorylation and translocation of BAD through a phosphatidylinositide3-OH kinase-dependent pathway. J Biol Chem 274:19411-19416.

Petros AM, Olejniczak ET, Fesik SW. 2004. Structural biology of the Bcl-2 family of proteins. Biochim Biophys Acta 1644:83-94.

Raman M, Chen W, Cobb MH. 2007. Differential regulation and properties of MAPKs. Oncogene 26:3100-3112.

Ramos JW. 2008. The regulation of extracellular signal-regulated kinase (ERK) in mammalian cells. Int J Biochem Cell Biol 40:2707-2719.

Rouleau C, Matécki S, Kalfa N, Costes V, de Santa Barbara P. 2009. Activation of MAP kinase (ERK1/2) in human neonatal colonic enteric nervous system. Neurogastroenterol Motil 21:207-214.

Sastry KS, Karpova Y, Kulik G. 2006. Epidermal growth factor protects prostate cancer cells from apoptosis by inducing BAD phosphorylation via redundant signaling pathways. J Biol Chem 281:27367-27377.

Scheid MP, Schubert KM, Duronio V. 1999. Regulation of bad phosphorylation and association with $\mathrm{Bcl}-\mathrm{x}(\mathrm{L})$ by the MAPK/Erk kinase. J Biol Chem 274:31108-31113.

Sharpe JC, Arnoult D, Youle RJ. 2004. Control of mitochondrial permeability by Bcl-2 family members. Biochim Biophys Acta 1644:107-113.

Shaul YD, Seger R. 2007. The MEK/ERK cascade: From signaling specificity to diverse functions. Biochim Biophys Acta 1773:1213-1226.
Shi J, Shen HM. 2008. Critical role of Bid and Bax in indirubin-3'-monoxime-induced apoptosis in human cancer cells. Biochem Pharmacol 75: 1729-1742.

Strasser A, O'Connor L, Dixit VM. 2000. Apoptosis signaling. Annu Rev Biochem 69:217-245.

Tafani M, Minchenko DA, Serroni A, Farber JL. 2001. Induction of the mitochondrial permeability transition mediates the killing of HeLa cells by staurosporine. Cancer Res 61:2459-2466.

Tzivion G, Shen YH, Zhu J. 2001. 14-3-3 proteins; bringing new definitions to scaffolding. Oncogene 20:6331-6338.

Weston CR, Davis RJ. 2007. The JNK signal transduction pathway. Curr Opin Cell Biol 19:142-149.

Wu J, Wong WW, Khosravi F, Minden MD, Penn LZ. 2004. Blocking the Raf/ MEK/ERK pathway sensitizes acute myelogenous leukemia cells to lovastatin-induced apoptosis. Cancer Res 64:6461-6468.

Yang R, Trevillyan JM. 2008. c-Jun N-terminal kinase pathways in diabetes. Int J Biochem Cell Biol 40:2702-2706.

Youle RJ, Strasser A. 2008. The BCL-2 protein family: Opposing activities that mediate cell death. Nat Rev Mol Cell Biol 9:47-59.

Zha J, Harada H, Yang E, Jockel J, Korsmeyer SJ. 1996. Serine phosphorylation of death agonist BAD in response to survival factor results in binding to 14-3-3 not BCL-X(L). Cell 87:619-628.

Zhang X, Li B, Song M, Song Y. 2004. Expression and significance of ERK protein in human breast carcinoma. Chin J Cancer Res 16:269273. 